\title{
Absolute Measurement of the Critical Behavior of the Smectic Elastic Constant of Bilayer and Monolayer Smectic-A Liquid Crystals on Approaching the Transition to the Nematic Phase
}

\section{Citation}

Fisch, M. R., Peter S. Pershan, and L. B. Sorensen. 1984. Absolute measurement of the critical behavior of the smectic elastic constant of bilayer and monolayer smectic-A liquid crystals on approaching the transition to the nematic phase. Physical Review A 29(5): 2741-2750.

\section{Published Version}

doi:10.1103/PhysRevA.29.2741

\section{Permanent link}

http://nrs.harvard.edu/urn-3:HUL.InstRepos:10361601

\section{Terms of Use}

This article was downloaded from Harvard University's DASH repository, and is made available under the terms and conditions applicable to Other Posted Material, as set forth at http:// nrs.harvard.edu/urn-3:HUL.InstRepos:dash.current.terms-of-use\#LAA

\section{Share Your Story}

The Harvard community has made this article openly available.

Please share how this access benefits you. Submit a story.

\section{Accessibility}




\title{
Absolute measurement of the critical behavior of the smectic elastic constant of bilayer and monolayer smectic- $A$ liquid crystals on approaching the transition to the nematic phase
}

\author{
M. R. Fisch, ${ }^{*}$ P. S. Pershan, and L. B. Sorensen ${ }^{\dagger}$ \\ Division of Applied Sciences, Harvard University, Cambridge, Massachusetts 02138
}

(Received 11 October 1983)

\begin{abstract}
A new technique which provides an absolute measurement of the smectic elastic constant $B$ is presented. This technique measures second sound on samples whose top surface is an air-liquidcrystal interface. The free surface of the sample is electrically driven and the resulting surface displacements are measured with an optical heterodyne technique. The smectic elastic constant is obtained from the spectrum of the scattered light which is determined by the bulk second-sound modes. Two bilayer and two monolayer smectic- $A$ liquid crystals were studied. Near the smectic$A$-to-nematic phase transition the data can be described by a simple power law $B=B_{1}\left(T-T_{N A}\right)^{\phi}$. However, the resulting critical exponents $\phi$ are not universal. In addition, the $B_{1}$ values for the bilayer smectics are considerably smaller than the values for the monolayer smectics.
\end{abstract}

\section{INTRODUCTION}

The nematic-to-smectic- $A$ phase transition remains one of the more intractable problems in equilibrium statistical physics. Following de Gennes's ${ }^{1}$ proposal in 1972 of a Landau theory in which the transition is analogous to the normal-to-superconducting transition, there have been a large number of experimental studies verifying the critical nature of the transition. However, the measured critical exponents do not display the universality that is expected from such a theoretical model. Furthermore, renormalization group arguments, including an anisotropic scaling hypothesis, ${ }^{2}$ result in specific relationships between these exponents that are only partially satisfied by the experimental results.

Halperin and Lubensky ${ }^{3}$ considered the effects that fluctuation phenomena should have on the smectic$A$-to-nematic transition and concluded that it should be weakly first order. A recent study of the superconducting transition by Dasgupta and Halperin ${ }^{4}$ that included the effects of fluctuations concluded that the smectic$A$-to-nematic transition should be continuous. Both light scattering ${ }^{5}$ and $x-$ ray $^{6}$ data indicate that to within a few millikelvins the transition appears continuous. Based on specific heat measurements Brisben et al. ${ }^{7}$ proposed that the transition might be close to a tricritical point and that the lack of universality could result from different materials being nearer or further from tricriticality. There is recent evidence supporting this hypothesis; ${ }^{8-10}$ however, quantitative discrepancies between the theory and experiment still exist. Nelson and Toner $^{11}$ recently proposed a model in which the transition from the smectic- $A$ to the nematic phase is mediated by the unbinding of dislocation pairs. They predict a specific relationship between the exponents which determine the critical temperature dependence of the longitudinal and transverse correlation lengths $\left(v_{\|}=2 v_{\perp}\right)$. Lubensky et al. ${ }^{12,13}$ have derived the same relation using a $1 / n$ expansion. Most of the existing experimental data violates the relationship $v_{\|}=2 v_{\perp}$.
The layered character of the smectic- $A$ phase gives rise to a bulk elastic modulus, $B$, that describes the elastic resistance to changes in the layer thickness at constant density. de Gennes has shown that $B$ is proportional to the square of the magnitude of the smectic order parameter, $\psi$. Since the various theories of the nematic-to-smectic- $A$ phase transition all make slightly different predictions for the critical temperature dependence of $B$, direct measurements of $B$ in the vicinity of the transition provide essential information from which different theories can be evaluated.

For $T<T_{N A}$ the superconducting analogy predicts a simple power law $B \sim\left(T_{N A}-T\right)^{\phi}$, with $\phi=0.67$ and $T_{N A}$ equal to the nematic-to-smectic- $A$ phase transition temperature. A weakly first order transition also exhibits a simple power law with $T_{N A}$ replaced by $T^{*}$, the temperature at which $B$ extrapolates to zero, and $\phi$ unspecified. The Nelson-Toner theory predicts that $B=B\left(T_{N A}\right)+$ "corrections to scaling" $\simeq B\left(T_{N A}\right)+B_{1}\left(T_{N A}-T\right)^{\phi}$. Almost all previous measurements show simple power-law behavior with $\phi$ between 0.25 and 0.6 (with a large cluster near $\frac{1}{3}$ ), and a transition that is continuous to within 10 $\mathrm{mK}$. However, our recent study of 80CB-60CB [4cyano-4'-(n-octyloxy)biphenyl and 4-cyano-4'-( $n$-hexyloxy)biphenyl] mixtures showed behavior consistent with the Nelson-Toner model. ${ }^{11}$

Most of the previous tests of the critical behavior of $B$ have been indirect in that they actually measure the length $\lambda=\sqrt{K_{1} / B}$. Although the Frank elastic modulus, $K_{1}$, is relatively independent of temperature near $T_{N A}$, it is very difficult to make a direct measurement of $K_{1}$ in a smectic- $A$ phase, and hence there is always some ambiguity in the value of $B$ obtained from these experiments. In these experiments $\lambda$ has been determined from either direct light scattering measurements ${ }^{14}$ or undulation instability measurements. ${ }^{15,16}$ Direct measurements of $B$ using low-frequency stress-strain techniques have also been performed; however, these techniques have not been employed to measure the critical behavior of $B$.

In principle $B / \rho$ can be determined directly from the 
speed of the shear or "second" sound wave. The mass density, $\rho$, has a small approximately linear, temperature dependence in the smectic- $A$ phase thus allowing simple calculations of $B$ from measured values of the speed. Unfortunately, conventional techniques are difficult to apply to second sound. For example, Brillouin scattering techniques $^{17}$ at gigahertz frequencies do not have sufficient resolution to obtain accurate values of $B$ as $B$ approaches zero. Furthermore, because of the high frequencies involved there may be dispersion in the elastic constants. There are also practical problems with conventional acoustic techniques. For example, since normal sound is 1 to 2 orders of magnitude faster than second sound, for a given frequency, the wavelength of normal sound is similarly larger. For a sample of fixed size it is exceedingly difficult to obtain a clean separation of the two signals. Standard acoustical techniques with second sound directly observed have only recently been developed. All previous ultrasonic studies inferred $B$ from small anisotropies in the velocity of conventional or "first" sound waves. ${ }^{18} \mathrm{Al}$ though Cheng et al. ${ }^{19}$ have recently observed second sound directly in a material with an exceptionally large $B$, it is doubtful that this technique could be applied to critical studies where $B \rightarrow 0$. Ricard and Prost ${ }^{20}$ measured $B$ on two materials using an interdigital electrode technique to excite second sound. Their technique has much in common with the technique to be described here and their results will be compared with ours below.

The paper presents direct measurements of the smectic elastic constant of four pure liquid crystals. We describe a new low-frequency technique developed for measuring second sound directly on samples whose top surface is a gas liquid-crystal interface. ${ }^{21,22}$ The material properties of the liquid crystal are determined from the experimentally measured distribution of light scattered by displacements of the free surface. This technique is analogous to measurements of capillary waves on nematic or isotropic samples. In contrast to capillary waves where surface displacements are dominated by surface tension, the surface deformations in a smectic- $A$ (at frequencies $\leqslant 1 \mathrm{MHz}$ and except very near zero frequency) are dominated by bulk elastic shear waves. Furthermore, since free surface samples should have fewer defects than confined samples, ${ }^{23}$ artifacts originating from sample imperfections should be significantly reduced in the present experimental geometry. Als-Nielsen, Christensen, and Pershan ${ }^{24}$ have carried out an x-ray diffraction study of smectic ordering at similar interfaces and found mosaic spreads less than $0.01^{\circ}$ over an area in excess of $1 \times 4 \mathrm{~mm}^{2}$. This is at least 2 orders of magnitude better alignment than is normally obtained in confined samples.

The remainder of this paper is divided into four parts. Section II outlines the theoretical basis of the present technique. Section III discusses the experimental aspects of this study. Section IV presents and discusses the results of this study. Section $V$ contains a summary of the present work.

\section{THEORETICAL PREDICTIONS}

In this section we outline the principal theoretical expressions for light scattering from a free surface smectic-
$A$ liquid-crystal sample. The relevant optical wave vectors and the experimental geometry are defined in Fig. 1. Assuming an incident wave vector $\overrightarrow{\mathrm{k}}_{I}$ there are the usual reflected $\left(\overrightarrow{\mathrm{k}}_{R}\right)$ and transmitted $\left(\overrightarrow{\mathrm{k}}_{T}\right)$ wave vectors and wave vectors $\overrightarrow{\mathrm{k}}_{S 1}$ and $\overrightarrow{\mathrm{k}}_{S 2}$ corresponding to waves scattered by surface displacements $u_{s}\left(\overrightarrow{\mathrm{q}}_{\perp}, \omega\right)$ at frequency $\omega$ and inplane wave vector

$$
\overrightarrow{\mathrm{q}}_{\perp}=\left(q_{x}, q_{y}\right)=\overrightarrow{\mathrm{k}}_{S(1,2)}-\overrightarrow{\mathrm{k}}_{I}
$$

For an incident wave polarized perpendicular to the plane of incidence and of unit magnitude one can demonstrate ${ }^{25}$ that in our experimental geometry where $\theta=\theta_{0} \simeq 45^{\circ}$ the wave scattered out of the sample has an amplitude

$$
E_{\text {scatt }} \simeq 2 i k_{0} \cos \theta R(\theta) u_{s}\left(\overrightarrow{\mathrm{q}}_{\perp}, \omega\right) \cos \phi,
$$

where $k_{0}=2 \pi / \lambda$, and $R(\theta)$ is the Fresnel reflection coefficient for perpendicular polarization. In the present experiment $\phi \simeq 10^{-2} \mathrm{rad}, \theta \simeq 45^{\circ}$, so that $q_{\perp} / k_{0} \ll 1$, $\cos \phi \simeq 1$, and $R(\theta)$ is essentially constant.

The scattered intensity $I_{\text {scatt }} \propto\left|E_{\text {scatt }}\right|^{2}$, therefore reduces to

$$
I_{\text {scatt }} \propto 4 k_{0}^{2} \cos ^{2} \theta R(\theta)^{2}\left|u_{s}\left(\overrightarrow{\mathrm{q}}_{\perp}, \omega\right)\right|^{2} .
$$

This is the essential result; note that it shows that the scattered light intensity depends on $\left|u_{s}\right|^{2}$ for small $u_{s}$. It also shows that the surface acts as a phase grating which, for small phase shifts, leads to only one scattered harmonic.

\section{A. Calculations of $u\left(\vec{q}_{\perp}, \omega\right)$}

The problem of scattering from a surface is thus reduced to finding $u_{s}\left(\overrightarrow{\mathrm{q}}_{1}, \omega\right)$. In order to do this the hydrodynamic equations describing the liquid crystal must be solved subject to appropriate boundary conditions. The hydrodynamics of a smectic- $A$ liquid crystal are described

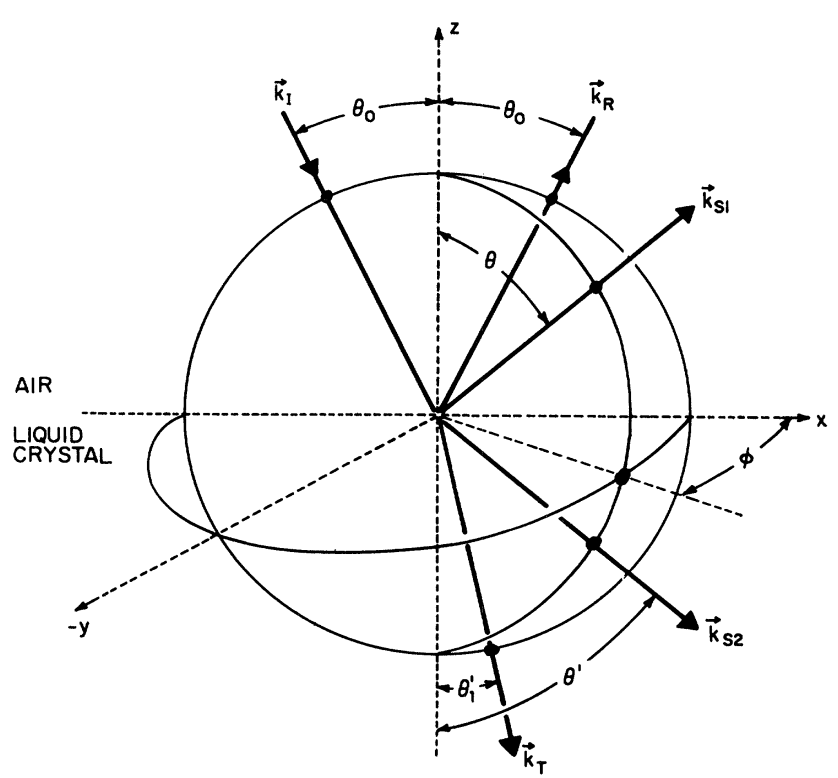

FIG. 1. Experimental geometry. $\left(\overrightarrow{\mathrm{k}}_{I}\right.$ is the incident wave vector, $\overrightarrow{\mathrm{k}}_{R}$ is the reflected wave vector, $\overrightarrow{\mathrm{k}}_{S 1}, \overrightarrow{\mathrm{k}}_{S 2}$ are the scattered wave vectors, and $\overrightarrow{\mathrm{k}}_{T}$ is the transmitted wave vector.) 
by six coupled equations, in six unknowns: $\rho, \overrightarrow{\mathrm{v}}, T$, and $u$; where $\rho$ is the density, $\overrightarrow{\mathrm{v}}$ the velocity field, $T$ the temperature, and $u$ the layer displacement. Assuming a plane wave of the form

$$
\overrightarrow{\mathrm{v}}(\omega, \overrightarrow{\mathrm{q}}) \propto \exp [i(\overrightarrow{\mathrm{q}} \cdot \overrightarrow{\mathrm{r}}-\omega t)]
$$

one of the six linear equations corresponds to an overdamped mode with $\vec{v}(\omega, \vec{q}) \cdot \vec{q}=0$ and $\vec{v}(\omega, \vec{q}) \cdot \hat{z}=0$ that does not induce surface displacements and which can be rigorously separated from the other equations. A second equation describing entropy or temperature fluctuations can be approximately separated. The other four equations are coupled together leading to a fourth order equation in $\omega$. Provided the damping is not too large this fourthorder becomes the product of two second-order equations,

$$
\left[\omega^{2}-2 i \omega v_{1} q^{2}-\frac{\beta}{\alpha}\right]\left[\omega^{2}-2 i \omega v_{2} q^{2}-\left(\alpha-\frac{\beta}{\alpha}\right)\right]=0,
$$

where $v_{1}$ is the effective viscosity for shear sound modes, $v_{2}$ is the effective viscosity for longitudinal sound modes,

$$
\begin{aligned}
& \alpha=C_{1} \rho q^{2}+\left(\frac{C_{2}}{\rho}-C_{3}\right) q_{z}^{2} \simeq C_{1} \rho q^{2}, \\
& \beta=\left(C_{1} C_{2}-C_{3}^{2}\right) q_{\perp}^{2} q_{z}^{2},
\end{aligned}
$$

and where second-order terms have been ignored and $C_{1}, C_{2}$, and $C_{3}$ are the elastic coefficients first introduced by de Gennes. ${ }^{26}$ The first term corresponds to an underdamped propagating shear mode (second sound), the second to an underdamped propagating longitudinal sound mode. The eigenfrequencies of the shear sound modes are much less than those of the longitudinal sound modes; typically $\beta / \alpha \simeq 10^{11} \mathrm{sec}^{-2}$, and $\alpha \approx 10^{14} \mathrm{sec}^{-2}$, thus $\omega_{\text {shear }} / \omega_{\text {long }} \approx 1 / 32$. Furthermore, since

$$
\delta \rho / \rho=\left(\omega_{\text {shear }} / \omega_{\text {long }}\right)^{2}=10^{-3},
$$

the quasishear mode may be treated as corresponding to a constant density, $\delta \rho=0$, mode. This mode depends on the material properties of interest and has the dispersion rela$\operatorname{tion}^{25}$

$\omega^{2}-2 i \omega v_{1 \text { eff }}\left(q_{\perp}^{2}+q_{z}^{2}\right)-\frac{B}{\rho} \frac{q_{\perp}^{2} q_{z}^{2}}{\left(q_{\perp}^{2}+q_{z}^{2}\right)}-\frac{K_{1}}{\rho} \frac{q_{\perp}^{6}}{\left(q_{\perp}^{2}+q_{z}^{2}\right)}=0$,

where as before $2 v_{1 \text { eff }}$ is the effective shear viscosity equal to $v, B$ is the smectic elastic constant approximately equal to $\beta / \alpha$, and $K_{1}$ is the Frank elastic constant.

In the present experiment $\overrightarrow{\mathrm{q}}_{\perp}$ is fixed by the incident and scattered wave vectors. The latter is determined by the position of the detector. The sample is driven at a specified frequency $\omega$ and the response calculated for fixed $\overrightarrow{\mathrm{q}}_{\perp}$ and $\omega$. For specified material parameters Eq. (4) is solved for $q_{z}$. Thus for given $\omega$ and $\vec{q}_{\perp}$ there are four roots for $q_{z}$ that come in complex conjugate pairs.

\section{B. Theoretical predictions for $q_{z}$}

The first solution is the conventional dispersion relation for undamped second sound:

$$
\left(q_{z}^{a}\right)^{2} \approx \frac{\omega^{2} q_{\perp}^{2}}{\frac{B}{\rho} q_{\perp}^{2}-\omega^{2}}
$$

or

$$
\omega^{2}=\frac{B}{\rho} \frac{q_{\perp}^{2} q_{z}^{2}}{\left(q_{\perp}^{2}+q_{z}^{2}\right)} .
$$

The second solution represents a boundary layer that has zero thickness if $v=0$

$$
\left(q_{z}^{b}\right)^{2} \approx \frac{i \omega}{v} \frac{q_{\perp}}{q_{z}^{a}}
$$

For typical experimental and material parameters $B \simeq 5 \times 10^{7} \mathrm{dyn} / \mathrm{cm}^{2}, q_{\perp}=q_{x}=125 \mathrm{~cm}^{-1}, q_{z}^{a}=50 \mathrm{~cm}^{-1}$, and $v=1 \mathrm{~cm}^{2} / \mathrm{sec}$, one obtains from Eq. (6) $\left|q_{z}^{b}\right|=1400$ $\mathrm{cm}^{-1}$; while the boundary layer is not totally negligible its effects are relatively small.

Of course, the above solutions do not represent the solution to the hydrodynamic problem in the experimental geometry. They must be supplemented by the proper boundary conditions. A simple set of approximate boundary conditions ${ }^{27}$ results from making the boundary layer given by Eq. (6) infinitely thin, or equivalently letting $v=0$.

For this boundary condition the response is a series of normal modes with

$$
\left(q_{z}^{a}\right)_{n}=(2 n+1) \frac{\pi}{2 h}, \quad n=0,1,2, \ldots
$$

where $h$ is the sample thickness. ${ }^{25}$

Figure 2 shows this solution to Eq. (4) including viscous effects in the propagating sound mode but neglecting the boundary layer. This clearly shows the dependence of the second sound resonant frequency on $q_{\perp}$ for various quantized $q_{z}$. It is important to observe that this mode is a bulk mode of the liquid crystal and not a surface mode. These modes are "waveguidelike" with a standing wave in

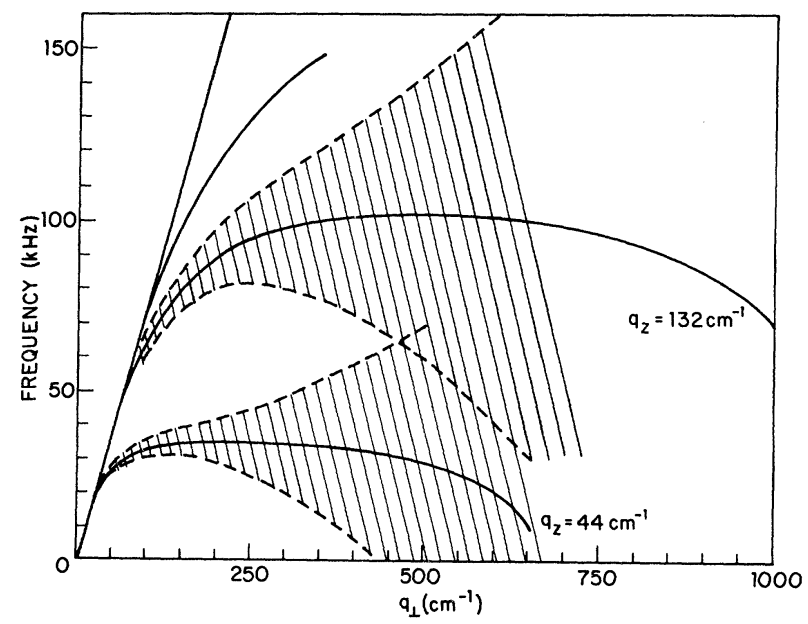

FIG. 2. Calculated second-sound frequencies as a function of $q_{\perp}$ for $B / \rho=2.5 \times 10^{7}(\mathrm{~cm} / \mathrm{sec})^{2}, v=1.0 \mathrm{~cm}^{2} / \mathrm{sec}$, and $h=360$ $\mu \mathrm{m}$. (Solid lines represent resonant frequency and dashed lines half-power points.) 
the $z$ direction and propagating waves in the $x$ direction. At large $q_{x}$ the viscous damping causes the solutions to become overdamped, and at small $q_{x}$ the boundary layer (ignored so far) becomes important. Nevertheless, there is a region over which underdamped propagating modes exist. Note also that the solution consists of well separated modes with an approximate resonant frequency

$$
\omega_{R}=\left(\frac{B}{\rho}\right)^{1 / 2} \frac{q_{x} q_{z}}{q},
$$

and a half-width $\Delta \omega=v_{1 \text { eff }} q^{2}$.

The above solution is correct for cases in which the inertial term equal to $\omega^{2}$ is comparable to or larger than the damping term equal to $2 i \omega v_{1 \text { eff }} q^{2}$. This is not the correct solution when the viscous damping term strongly dominates the inertial term,

$$
\frac{\omega}{v_{1 \mathrm{eff}} q^{2}}<<1
$$

In this region the Frank modulus term must be kept and the solution is identical to that of the Orsay group: $:^{28}$

$$
i q_{z}=q_{z}^{2}\left(\frac{K}{B}+\frac{i \omega v_{1 \mathrm{eff}}}{B q_{x}^{2}}\right)^{1 / 2} .
$$

This overdamped mode is centered at zero frequency and has a half-width $\lesssim 1 \mathrm{~Hz}$; it is the surface wave of the present system. We did not study this mode.

The amplitude of the bulk layer displacement $u(\overrightarrow{\mathrm{q}}, \omega)$ must be related to the surface displacement $u_{s}\left(q_{\perp}, \omega\right)$. Assuming that the bulk modes are underdamped and uncoupled the surface displacement can be calculated as

$$
u_{s}\left(q_{\perp}, \omega\right)=\sum_{n=1}^{N} u\left(q_{\perp}, q_{z}(n), \omega\right),
$$

where $N$ is some large number that represents the highfrequency or high-wave-vector cutoff of our approximations. For a mode near resonance the resonant mode will dominate and

$$
u_{s}\left(q_{\perp}, \omega\right) \simeq u\left(q_{\perp}, q_{z}, \omega\left(q_{\perp}, q_{z}\right)\right)
$$

where $\omega\left(q_{\perp}, q_{z}\right)$ is given approximately by Eq. (5).

This result allows the surface displacement to be calculated for a number of cases. The case of thermal excitation has been previously considered. ${ }^{21}$ It was shown that thermally induced waves are too weak to be readily measured. Following the approach of Sohl et al. ${ }^{29}$ the top surface of the sample is excited at a single scanned frequency. The resulting surface displacement may be found for both $v \rightarrow 0$ and more realistic boundary conditions.

The comparison between the experimentally measured spectrum and the $v \rightarrow 0$ theory just presented are shown in Figs. 3(a) and 3(b). For the typical response shown in Fig. 3(b) $N$ is 15 . For most of the curves fit to this form the best fit resulted from $N$ in the range 5-15. This follows because as $n$ increases $q_{z}$ becomes larger and since

$$
\left(\overrightarrow{\mathrm{q}}=\hat{\perp} q_{\perp}+\hat{z} q_{z}\right) \cdot \overrightarrow{\mathrm{u}} \simeq 0,
$$

the displacements caused by the second sound mode become parallel to the surface and do not scatter light.
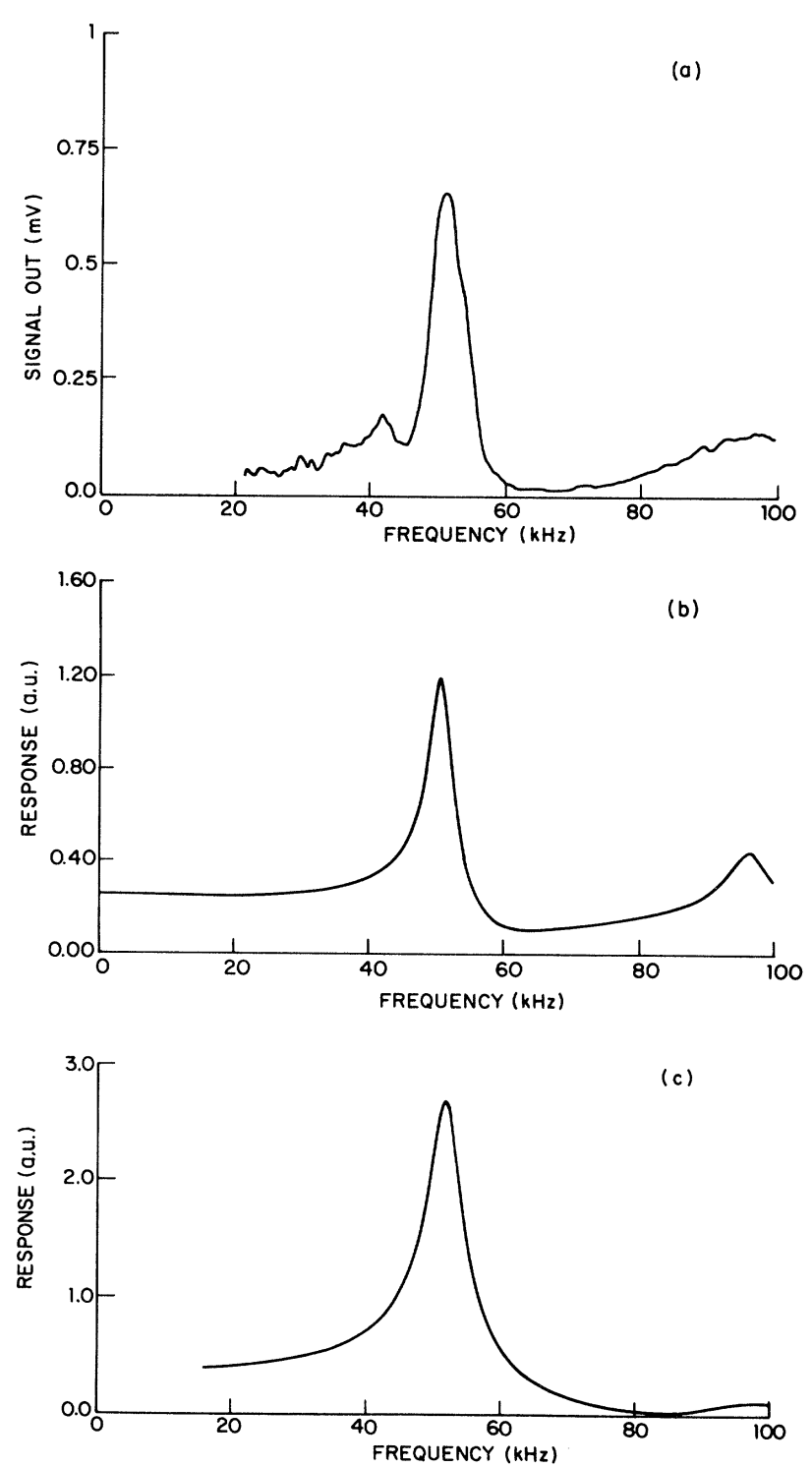

FIG. 3. Comparison of experimentally measured response to theoretical models. (a) Experimentally measured response for 8OCB sample: $q_{z}=60 \mathrm{~cm}^{-1}, q_{x}=125 \mathrm{~cm}^{-1}, \Delta T=0.14 \mathrm{~K}$. (b) Prediction of simple theory that ignores the boundary layer: $B=3.51 \times 10^{7} \mathrm{dyn} / \mathrm{cm}^{2}, \quad \eta=1.5 \mathrm{P}$ (independent of angle), $\rho=1.0 \mathrm{~g} \mathrm{~cm}^{-3}, q_{x}=125 \mathrm{~cm}^{-1}, q_{z}=60 \mathrm{~cm}^{-1}(h=262 \mu \mathrm{m})$. (c) Prediction of theory with boundary layer included: $B=3.51 \times 10^{7} \mathrm{dyn} / \mathrm{cm}^{2}, \quad \eta=0.9 P$ (independent of angle), $\rho=1.0 \mathrm{~g} \mathrm{~cm}^{-3}, q_{x}=125 \mathrm{~cm}^{-1}, q_{z}=60 \mathrm{~cm}^{-1}$.

Although the simple boundary conditions just described fit the experimental data quite well a more complete theory, including the effects of the boundary layer, was explored to ensure the fundamental correctness of the approach just presented. The results of this approach are presented by Fisch. ${ }^{25}$ The basic result is that for a given $\omega$ and $q_{x}$ and sample thickness the same resonant frequency $( \pm 1 \%)$ is predicted as in the simple theory. The half-widths of the resonance peaks are somewhat larger in this theory; this leads to an effective viscosity that is approximately $\frac{2}{3}$ that of the simple theory when curve fitting is done. However, since $B$ is determined from the 
resonant frequencies of the modes this small difference is not important

\section{EXPERIMENTAL APPARATUS AND PROCEDURES}

A diagram of the experimental apparatus is shown in Fig. 4. This system may be conveniently broken into four component parts: the laser and associated optical components, the sample exciter or driver, the electronics for both the driver and the detector circuit, and the ovensample holder. Each of these parts will be discussed in the following. The optical system begins with a Coherent Radiation, Inc. model CR-52 argon-ion laser operating at $5145 \AA$. To ensure the absence of unwanted heating effects the laser power is reduced with neutral density filters to $\lesssim 5 \mathrm{~mW}$. Furthermore, the laser polarization is rotated by a $\lambda / 2$ plate into the horizontal plane. This reduces any Brewster angle effects that might occur at the liquidcrystal surface. The polarization is further defined to $10^{-5}$ by a Glan-Thompson polarizer. The light is weakly focused onto the sample as shown in the figure.

A heterodyne detection scheme similar to that developed by Hard et al. ${ }^{30}$ is used in the present experiment. In the present heterodyne detection scheme high resolution is obtained by mixing [on the photomultiplier tube (PMT) photocathode] the Doppler-shifted scattered light with a small fraction $\left(\simeq 10^{-6}\right)$ of the light specularly reflected from the sample surface. The local oscillator is provided by a selected diffractive order of the grating placed after the sample. The spacing of the grating used in the present study leads to a $q_{\perp}$ that is any one of the lower harmonics of $125 \mathrm{~cm}^{-1}$. The sensitivity of the present system is quite high. Thermally excited surface waves on nematic liquid crystals with a theoretically predicted rms displacement of $0.01 \AA$ are detected with a signal to noise of 2:1 in one 4-sec scan of a Tektronics 7L5 spectrum analyzer. The advantages of such a system and further details are described by Fisch $^{25}$ elsewhere. The optical system is completed by the optical bandpass filter that removes light more than a few angstroms from the incident wavelength and an RCA 7265 PMT.

The device used to drive the surface is similar to one designed by Sohl, Ketterson, and Miyano. ${ }^{29}$ It is based on the well-known fact that dielectrics in an electric field deform so as to minimize the total (elastic and electromagnetic) energy of the system. In the present experiment an

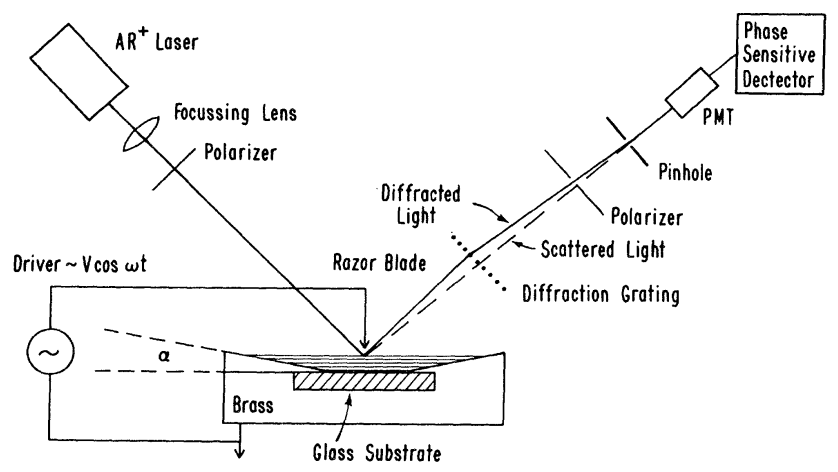

FIG. 4. Diagram of experimental apparatus.

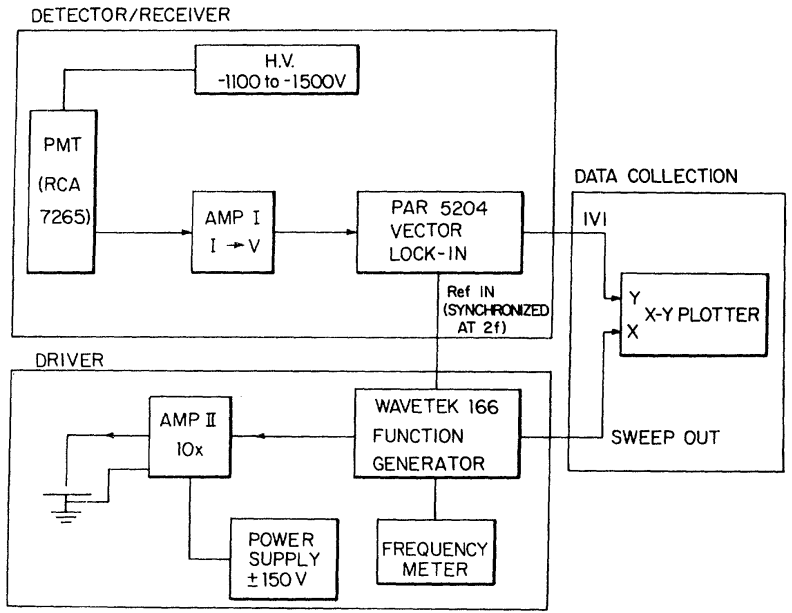

FIG. 5. Block diagram of electronics.

ac voltage (typically between $40-125 \mathrm{~V}$ ) is applied between a knife edge (approximately $25 \mu \mathrm{m}$ above the top of the sample) and a conducting glass substrate on which the sample rests. Although the general solution to the elastic and electrodynamic problem is complex, linear-response theory requires that the oscillatory part of the surface displacement, $u_{s}\left(q_{\perp}, \omega\right)$, be proportional to $v_{0}^{2} \cos 2 \omega_{D} t$, where $v_{0}$ is the peak voltage applied between the knife edge and the conducting substrate, and $\omega_{D}$ the angular frequency of the driver. It was experimentally verified that the amplitude of the response was proportional to $v_{0}^{2}$ and that the response was indeed at $2 \omega_{D}$.

A block diagram of the electronic system shown in Fig. 5 is of conventional design. The receiver circuit begins

(a)

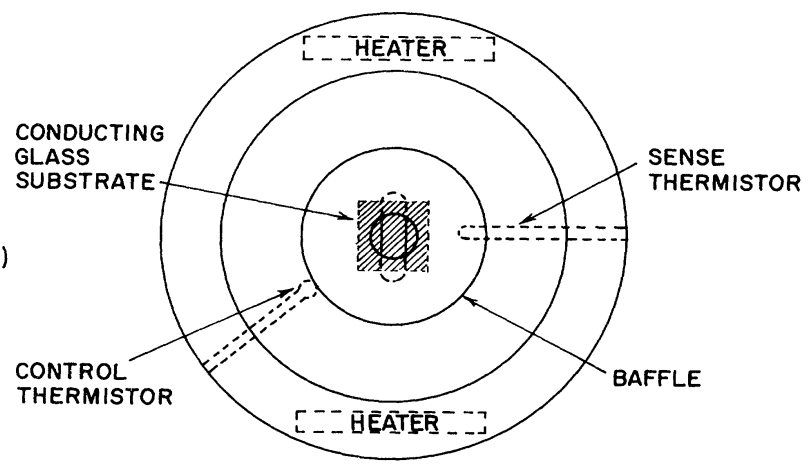

(b)

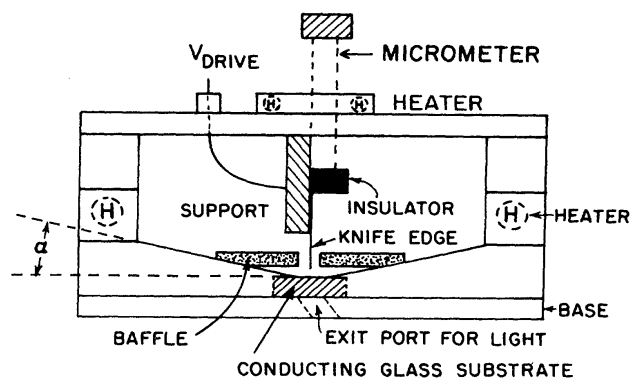

FIG. 6. Details of the oven. (a) Top view of oven bottom plate-sample holder. (b) Cross section of oven; $\alpha$ is the contact angle. 
TABLE I. Liquid crystals used in the present study.

\begin{tabular}{|c|c|c|c|c|c|}
\hline $\begin{array}{l}\text { Liquid crystal name and } \\
\text { chemical formula }\end{array}$ & Acronym & $\begin{array}{r}\text { Transition } \\
\text { temperat }\end{array}$ & & $T_{N A} / T_{N I}$ & Source \\
\hline 4-cyano-4'-(n-octyloxy) biphenyl & $8 \mathrm{OCB}$ & $\begin{array}{l}\text { Crystal } \rightarrow \operatorname{Sm} A \\
\operatorname{Sm} A \rightarrow N \\
N \rightarrow I\end{array}$ & $\begin{array}{l}54.5 \\
67.1 \\
80\end{array}$ & 0.963 & $\begin{array}{l}\text { BDH Chemical Ltd. } \\
\text { Poole, England }\end{array}$ \\
\hline 4-cyano-4'-(n-octyl) biphenyl & $8 \mathrm{CB}$ & $\begin{array}{l}\text { Crystal } \rightarrow \operatorname{Sm} A \\
\operatorname{Sm} A \rightarrow N \\
N \rightarrow I\end{array}$ & $\begin{array}{l}21.5 \\
33.5 \\
40.5\end{array}$ & 0.978 & BDH Chemicals Ltd. \\
\hline 4-( $n$-butyloxy) benzylidene-4'-(n-octyl) aniline & 40.8 & $\begin{array}{l}\text { Crystal } \rightarrow \operatorname{Sm} B \\
\mathrm{Sm} B \rightarrow \operatorname{Sm} A \\
\operatorname{Sm} A \rightarrow N \\
N \rightarrow I\end{array}$ & $\begin{array}{l}33 \\
49.5 \\
63.5 \\
79.0\end{array}$ & 0.956 & $\begin{array}{l}\text { Organix } \\
\text { Avon, N.Y. }\end{array}$ \\
\hline 4-( $n$-pentylphenylthio)-4'-(n-octyloxy) benzoate & $\overline{8} \mathrm{~S} 5$ & $\begin{array}{l}S C \rightarrow \operatorname{Sm} A \\
\operatorname{Sm} A \rightarrow N \\
N \rightarrow I\end{array}$ & $\begin{array}{l}55.55 \\
63.05 \\
86.35\end{array}$ & 0.935 & $\begin{array}{l}\text { M. Neubert } \\
\text { Liquid Crystal } \\
\text { Institute, Kent State }\end{array}$ \\
\hline
\end{tabular}

with the PMT. The current output of this device is fed into AmpI that acts as a current-to-voltage converter followed by a high-pass filter. The output of the $I \rightarrow V$ is fed into a Princeton Applied Research PAR-5204 phase sensitive detector amplifier. This instrument has features that enable the internal local oscillator to remain phaselocked to the second harmonic of a variable frequency external reference as the latter is swept. The magnitude of the output signal is connected to the $y$ input of the $x-y$ recorder.

The driver circuit begins with a Wavetek 166 Function Generator. Its output is continuously adjustable up to maximum output of $15-\mathrm{V}$ peak. This signal is amplified using an Analog Devices, Inc. 171-K amplifier that is wired as a $10 \times$ amplifier.

The Wavetek 166 has an internal ramp generator that linearly $( \pm 1 \%)$ sweeps the frequency and provides an output voltage proportional to the frequency. This voltage is connected to the $x$ input of the $x-y$ recorder. This feature is used to collect the data by generating curves of the volt- age output from the lock-in versus frequency. The peaks in such curves represent the resonant frequencies of the light scattered from the sample. Because the signal output is proportional to the photocurrent induced by heterodyning the scattered light and not the scattered power the 0.707 points of such peaks determine $\Delta \omega$ and are related to the viscosity of the liquid crystal and other damping processes.

A drawing of the oven is shown in Fig. 6. It consists of a polished brass side-wall plate that is machined to the contact angle $\alpha$ of the brass-liquid crystal-air interface. This angle is approximately $11^{\circ}$ but varied somewhat from material to material. By varying the contact angle, convex and concave samples as well as the desired flat samples could be produced. A conducting glass substrate is inserted into the bottom center of this plate, forming a sample holder for creating free surface samples. As shown in the figure there are both heated side walls and a top plate (with the micrometer upon which the knife edge is mounted) on top of the sample holder.

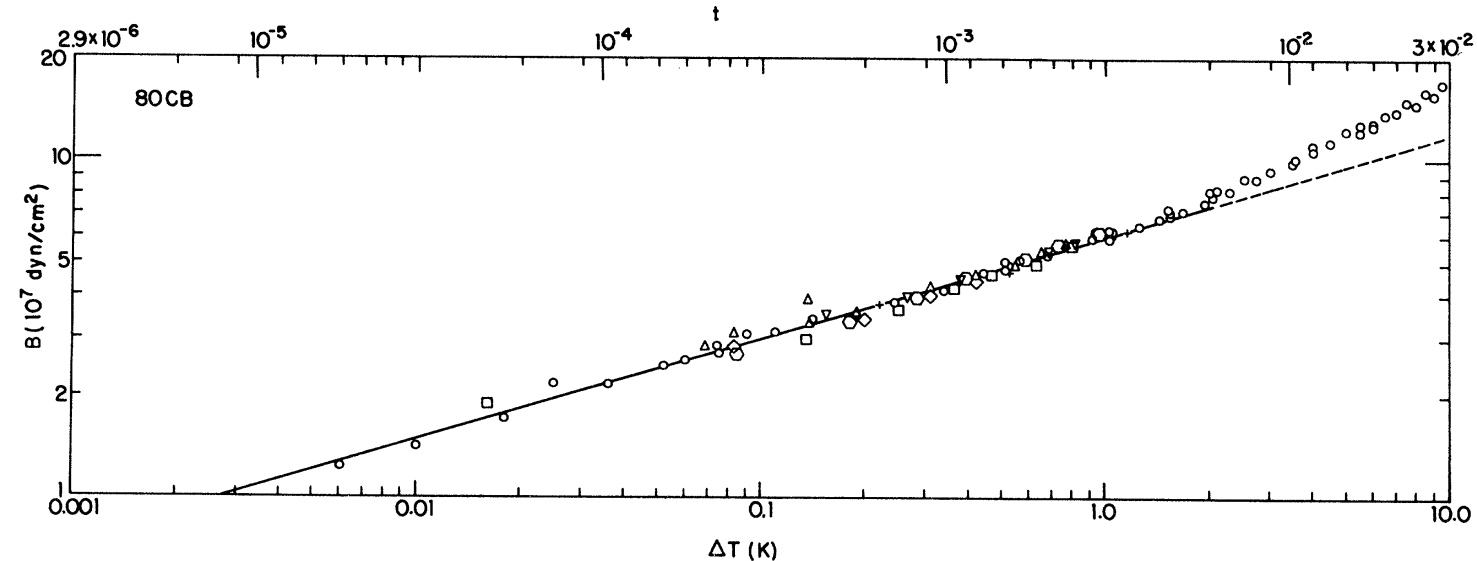

FIG. 7. $B(\Delta T)$ vs $\Delta T$ for 8OCB. ( $\square: q_{z}=112 \mathrm{~cm}^{-1}, q_{x}=125 \mathrm{~cm}^{-1} ; \Delta: q_{z}=55 \mathrm{~cm}^{-1}, q_{x}=125 \mathrm{~cm}^{-1} ; \diamond: q_{z}=55 \mathrm{~cm}^{-1}, q_{x}=250$ $\mathrm{cm}^{-1} ;+: q_{z}=55 \mathrm{~cm}^{-1}, q_{x}=375 \mathrm{~cm}^{-1} ; \nabla: q_{z}=60 \mathrm{~cm}^{-1}, q_{x}=125 \mathrm{~cm}^{-1} ; 0: q_{z}=33.4 \mathrm{~cm}^{-1}, q_{x}=125 \mathrm{~cm}^{-1} ; \circ: q_{z}=38.9 \mathrm{~cm}^{-1}$, $q_{x}=125 \mathrm{~cm}^{-1}$.) 


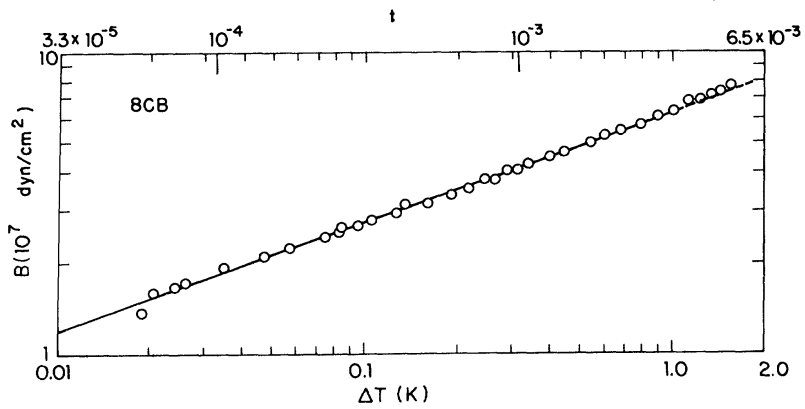

FIG. 8. $B(\Delta T)$ vs $\Delta T$ for $80 C B, q_{z}=30.25 \mathrm{~cm}^{-1}, q_{x}=125$ $\mathrm{cm}^{-1}$.

In order to reduce temperature gradients and to help break up convective flow within the oven a copper baffle is placed on top of the bottom plate. The temperature is controlled by a Yellow Springs Instrument Co., Inc. model 72 temperature controller.

This oven assembly is enclosed by insulation and mounted on an adjustable plate so that the oven can be leveled. An outer can is mounted on top of this assembly to further isolate the oven from room temperature fluctuations. The short-term temperature stability is on the order of $2 \mathrm{mK}$. The long-term stability depended on variations in room temperature but was approximately 10 $\mathrm{mK}$. There is some evidence for small temperature gradients within the sample; these are largest near the knife edge and typically were about $5 \mathrm{mK}$. The temperature homogeneity improved rapidly in regions away from the knife edge. Even at $T_{N A}-T=10 \mathrm{mK}$ several spots on the sample yielded the same value of the smectic elastic coefficient indicating that these gradients are not important over the temperature range of most of the data.

Sample preparation and experimental procedure

The oven is prepared to form free surface samples by coating it with the surfactant HTAB (hexadecyltrimethylammonium bromide). The oven is then taken to a temperature at which the sample will be in its isotropic phase and the liquid crystal added to the oven. Typically 0.2-0.3 grams of material were required. Special care was exercised to ensure that the bottom and sides of the oven were coated with liquid crystal. Well-aligned sam-

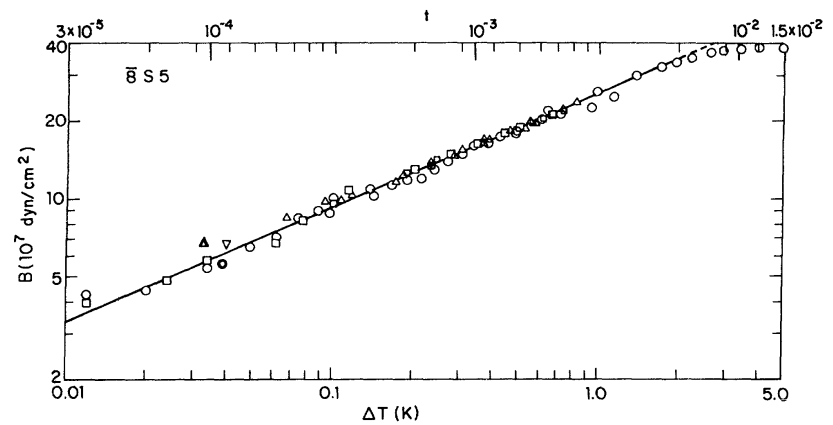

FIG. 9. $B(\Delta T)$ vs $\Delta T$ for $\overline{8} \mathrm{~S} 5$. $\left(\triangle ; q_{z}=45.4 \mathrm{~cm}^{-1}, q_{x}=125\right.$ $\mathrm{cm}^{-1} ;$ O $: q_{z}=34.4 \mathrm{~cm}^{-1}, q_{x}=125 \mathrm{~cm}^{-1}, \square: q_{z}=27.8 \mathrm{~cm}^{-1}$, $q_{x}=125 \mathrm{~cm}^{-1} ; \nabla: q_{z}=60.2 \mathrm{~cm}^{-1}, q_{x}=125 \mathrm{~cm}^{-1}$.)

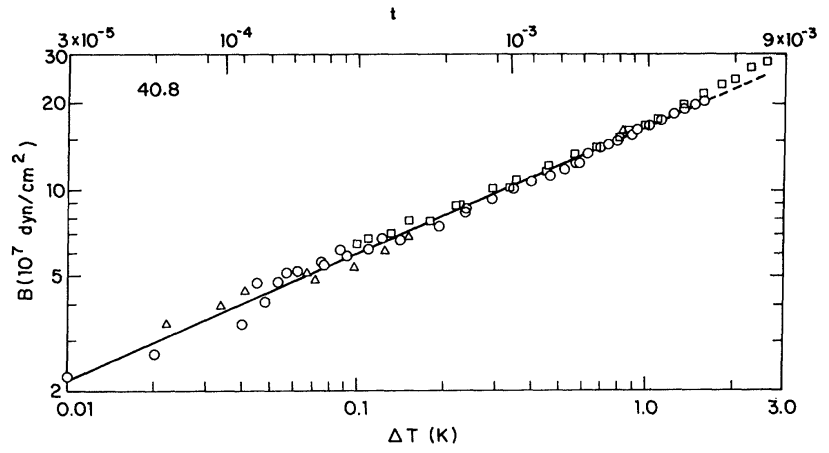

FIG. 10. $B(\Delta T)$ vs $\Delta T$ for $40.8\left(\Delta: q_{z}=35.7 \mathrm{~cm}^{-1}\right.$, $q_{x}=125 \mathrm{~cm}^{-1} ; \square: q_{z}=41.3 \mathrm{~cm}^{-1}, q_{x}=125 \mathrm{~cm}^{-1} ; \bigcirc^{\prime} q_{z}=29.7$ $\mathrm{cm}^{-1}, q_{x}=125 \mathrm{~cm}^{-1}$.).

ples generally resulted from several heating and cooling cycles between the smectic- $A$ and isotropic phases. On occasion this had to be supplemented with either stirring and/or cooling to room temperature. ${ }^{25}$ The details of the procedure used to obtain well aligned samples did not affect the measured elastic properties of the sample.

The transition temperature $T_{N A}$ was determined by observing the disappearance of the large nematic response to an applied electric field. This could easily be recognized since the response of the nematic free surface is at least 2 to 3 orders of magnitude larger than that of the smectic surface. The temperature measured in this way agrees to within $5 \mathrm{mK}$ of the temperature measured by looking for the disappearance of the smectic resonances on heating. The transition temperature was measured daily to test for any drift. For 40.8 which is much less stable than the other compounds studied the transition temperature was also measured at the end of a day's run. In the presence of a small positive pressure of either argon or dry nitrogen the drift in $T_{N A}$ was less than $0.005^{\circ} \mathrm{C} /$ day for 40.8 and nonmeasurable for the others. Sample quality was monitored during the course of the experiment and only data from well-aligned samples is reported.

The data consisted of a series of spectra for various $T_{N A}-T, q_{z}$, and $q_{x}$. The resonant frequency of the modes was extracted and related to $B$ using the relation

$$
\omega_{0} \simeq\left(\frac{B}{\rho}\right)^{1 / 2} q_{x} q_{z}\left(q_{x}^{2}+q_{z}^{2}\right)^{-1 / 2}
$$

Corrections were made for the effect of damping on the resonant frequency; however, these are small (1-2)\% effects. The resonant frequency of the peaks is very reproducible both from sample to sample and day to day. The damping data is not so straightforward. Samples that were not very flat scattered less strongly and had a larger apparent viscosity than flat samples. For this reason there was sometimes variation (in half-width but not in peak position) from point to point on the same sample. Although systematic interpretation of the linewidth data is not warranted it is clear that good samples will obtain a linewidth corresponding to an effective viscosity of less than $1.5 \mathrm{P}$, and there is no apparent divergence in this value near the transition temperature. The data for $80 \mathrm{CB}$ indicates that there is a systematic decrease in the viscosi- 
TABLE II. Results in pure compounds. Results of present study. $B_{1}$ and $B_{1}^{\prime}$ in units of $10^{7}$ dyn $/ \mathrm{cm}^{2} \mathrm{~K}^{\phi}, B\left(T_{N A}\right)$ in units of $10^{7} \mathrm{dyn} / \mathrm{cm}^{2}$.

\begin{tabular}{lccccc}
\hline \hline Material & \multicolumn{2}{c}{$B\left(T_{N A}\right)=0$} & \multicolumn{3}{c}{$B\left(T_{N A}\right) \neq 0$} \\
& $B_{1}$ & \multicolumn{1}{c}{$\phi$} & $B\left(T_{N A}\right)$ & $B_{1}$ & \multicolumn{1}{c}{$\phi$} \\
\hline 8 8CCB & $5.96 \pm 0.12$ & $0.30 \pm 0.023$ & $0.15 \pm 0.43$ & $5.9 \pm 0.43$ & $0.33 \pm 0.04$ \\
$8 \mathrm{CB}$ & $6.22 \pm 0.12$ & $0.36 \pm 0.03$ & $0.07 \pm 0.34$ & $6.31 \pm 0.31$ & $0.37 \pm 0.034$ \\
40.8 & $16.7 \pm 0.34$ & $0.44 \pm 0.025$ & $0 \pm 1.0$ & $16.6 \pm 0.8$ & $0.44 \pm 0.04$ \\
$\overline{8 S 5}$ & $25.3 \pm 0.5$ & $0.44 \pm 0.025$ & $0 \pm 1.4$ & $25.3 \pm 1.2$ & $0.44 \pm 0.04$ \\
\hline \hline
\end{tabular}

ty at higher temperatures just as expected. The sample height was measured after a run by covering the top of the sample with grit and measuring the height under a microscope. This technique agreed very well with the height as determined by the ratio of the resonant frequency of the first two modes, and was essentially in agreement with the crude estimate that could be obtained from the position of the driver electrode. The height was measured to $\pm 10 \mu$ by the grit technique and $(2-3) \%$ by the ratio of the resonant frequencies technique. These represent approximately equivalent errors $( \pm 5 \%)$ in $B$.

\section{RESULTS}

Four pure liquid crystals were investigated; the chemical name, common acronym, chemical formula, and phase transition temperature of each of these materials are shown in Table I. Two types of materials were studied: monolayer smectics, where each smectic layer contains one molecule [4-( $n$-butyloxy) benzylidene- 4 '-( $n$-octyl) aniline (40.8) and 4-(n-pentylphenylthio)-4'-(n-octyloxy)benzoate $(\overline{8} \mathbf{S} 5)]$, and bilayer smectics where each smectic layer contains two molecules [8OCB and 4cyano-4'-( $n$-octyl)biphenyl (8CB)]. The most extensively studied material was $80 \mathrm{CB}$. This material was chosen for extensive study because it is very stable and its smectic$A$-to-nematic phase transition has been one of the most studied.

The results indicate that if the data for $B$ is fit to the form $B=B_{1}\left(T_{N A}-T\right)^{\phi}$ the effective critical exponent $\phi$ is not universal. Graphs of the measured values of $B$ vs $\Delta T=T_{N A}-T$ for the four liquid crystals are shown in Figs. 7-10. The reduced temperature scale, $t=\left(T_{N A}-T\right) / T_{N A}$, is displayed on the top of each figure. The parameters of various fits are tabulated in Table II. For both $80 \mathrm{CB}$ and $8 \mathrm{CB} B$ was extracted from the measured quantity $B / \rho$ using published density measure- ments. $^{31,32}$ For $\overline{8}$ S5 and 40.8 we assumed that $\rho=1.0$ $\mathrm{g} / \mathrm{cm}^{3}$. Although the transition temperature $T_{N A}$ varied slightly from sample to sample for each liquid crystal, the $B(\Delta T)$ for any given material curves did not depend on $T_{N A}$. In all cases the transition appeared second order to within $5 \mathrm{mK}$. The data for $\Delta T \lesssim 1.5 \mathrm{~K}$ in all samples could be represented by the simple power law: $B=B_{1}\left(T_{N A}-T\right)^{\phi}$. The nonlinear least squares fits of the data to this form are shown by the solid lines in the figures. Their extrapolation to larger $\Delta T$ is indicated by the broken line. In all cases the normalized $\chi^{2}$ was essentially 1 , and the largest part of the error in $B_{1}$ was due to the uncertainty in measuring the sample height. It is clear from the results tabulated in Table II that $B_{1}$ is much smaller for the bilayer smectics than the monolayer smectics and also that $\phi$ is substantially larger for the monolayer smectics. The data was also fit to the form

$$
B=B\left(T_{N A}\right)+B_{1}^{\prime}(\Delta T)^{\phi}
$$

thus allowing for the possibility that $B \neq 0$ at $T_{N A}$. The results of least square fits to the data are also tabulated in Table II. These fits had $\chi^{2}$ 's that were essentially the same as those in which $B\left(T_{N A}\right) \equiv 0$. Finally previous published results ${ }^{22}$ indicate that the present technique measures the true hydrodynamic behavior of the liquid crystals.

\section{DISCUSSION}

Several conclusions may be drawn from the results summarized in Table II. Firstly, the exponent $\phi$ is not universal; in fact, it appears to be bimodal. The bilayer smectics (8CB and 8OCB) have an apparent exponent of approximately 0.33 . The monolayer smectics $(\overline{8} \mathrm{~S} 5$ and 40.8) have an apparent exponent of 0.44. Furthermore,

TABLE III. Results in pure compounds. Results of other studies. Numbers in parentheses are reference numbers.

\begin{tabular}{lcccccc}
\hline \hline Exponent & $v_{\|}^{N}$ & $v_{\perp}^{N}$ & $\gamma^{N}$ & $\alpha$ & $v_{\|}^{\mathrm{Sm}}$ & $\phi$ \\
Material & $\xi_{\| \sim(\Delta T)^{-v_{\|}}}$ & $\xi_{\perp} \sim(\Delta T)^{-v_{\perp}}$ & $\chi \sim(\Delta T)^{-\gamma}$ & $c_{v} \sim(\Delta T)^{-\alpha}$ & $\xi_{\| \sim(\Delta T)^{-v_{\|}}}$ & $B \sim(\Delta T) \phi$ \\
\hline 8 OCB & $0.71 \pm 0.04$ & $0.58 \pm 0.04$ & $1.32 \pm 0.06$ & $0.23 \pm 0.04$ & $0.5 \pm 0.03$ & $0.30 \pm 0.05$ \\
& $(6)$ & $(6)$ & $(6)$ & $(6)$ & $(6)$ & $(6)$ \\
$8 \mathrm{CB}$ & $0.67 \pm 0.02$ & $0.51 \pm 0.02$ & $1.26 \pm 0.06$ & & & $0.26 \pm 0.06$ \\
& $(6)$ & $(6)$ & $(6)$ & & $(6)$ \\
40.8 & $0.71 \pm 0.01$ & $0.57 \pm 0.01$ & $1.31 \pm 0.02$ & $0.15 \pm 0.05$ & $0.54 \pm 0.04$ & $0.32 \pm 0.03$ \\
& $(34)$ & $(34)$ & $(34)$ & $(34)$ & $(35)$ & $(34)$ \\
$\overline{8 S 5}$ & 0.83 & 0.68 & 1.53 & $0(\log )$ & & \\
& $(36)$ & $(36)$ & $(36)$ & $(37)$ & & \\
\hline \hline
\end{tabular}


the exponent $\phi$ exhibits a systematic decrease as $T_{N A} / T_{N I}$ increases $\left(T_{N I}\right.$ is the isotropic $\rightarrow$ nematic phase transition temperature). Similar systematic variations of the other critical exponents with $T_{N A} / T_{N I}$ have been observed. ${ }^{36-38}$ These systematic trends predominate over the bilayer-monolayer nature of the substance. ${ }^{36,37}$ Secondly, the elastic modulus, and hence also the magnitude of the smectic order parameter squared is three to four times larger in the monolayer smectics than in bilayer smectics at the same $\Delta T$. There is no theory that explains this difference in the magnitude of the smectic elastic constant.

A direct measurement of $B$ in $80 C B$ and $8 C B$ has been performed by Ricard and Prost ${ }^{20}$ using an interdigital electrode technique. Their results for $80 \mathrm{CB}$ are indistinguishable from our own. However, they find an exponent $\phi \simeq 0.49$. This is because they treated the transition as being weakly first order, and fit their data over the temperature range $0.065<\Delta T<6.1 \mathrm{~K}$ with $T_{N A}-T^{*}=100 \mathrm{mK}$. It is clear from Fig. 7 that there is an enhancement in the measured $B$ above its predicted simple power-law value for $\Delta T<1.5 \mathrm{~K}$. Our value of $\phi$ also agrees with the value obtained from measurements of $\lambda=\sqrt{K_{1} / B}$ by Birecki et al. ${ }^{14}$ From their measurement of $\lambda$ we infer a value of $K_{1}$ of $1.6 \times 10^{-6}$ dyn $( \pm 20 \%)$; this is in good agreement with the value $1.8 \times 10^{-6}$ dyn obtained by Karat and Madhusindance. $^{33}$ In $8 \mathrm{CB}$ Ricard and Prost obtain $\phi \simeq 0.63$ and $T_{N A}-T^{*}<10 \mathrm{mK}$. However, in this material their data is quite different from ours, yielding the same value of $B$ only at $\Delta T \simeq 1 \quad \mathrm{~K}$; falling below our data by increasingly larger amounts as $\Delta T \rightarrow 0$. Furthermore, the effective viscosity they obtain is five times larger than that obtained in $8 \mathrm{OCB}$, our results yield very similar viscosities with $v_{8 \mathrm{CB}} / v_{8 \mathrm{OCB}} \simeq 1$. The origin of this disagreement is unclear at present.

Not surprisingly this nonuniversality also appears in the other parameters that exhibit critical phenomena; some of these parameters are shown in Table III. The final column reports the value of $\phi$ measured by other techniques, for the bilayer smectics these measurements are in agreement with those measured in the present study; however, the value of $\phi$ in 40.8 is quite different from that measured with the present technique. This problem is further emphasized by comparing the values of various critical exponents, as predicted by the scaling relations, with those actually measured. The two relations that shall be used were predicted by Lubensky and Chen ${ }^{2}$ and are (a) $\phi=2 v_{\perp}-v_{\|}$and (b) $v_{\|}+2 v_{\perp}=2-\alpha$, where $\alpha$ is the exponent describing the divergence of the specific heat. The results of these calculations are shown in Table IV. The simplest test uses relation (a) to calculate $\phi$ from the measured nematic values of $v_{\|}^{N}$ and $v_{\perp}^{N}$. These values are in rough agreement with the data from the present study. A second test obtains $\nu_{\perp}^{\mathrm{Sm}}$ (where the superscript $\mathrm{Sm}$ means in the smectic phase) from relation (b) and the measured values of $\alpha$ and $v_{\|}^{\mathrm{Sm}}$, and then obtains $\phi$ from relation (a). The values obtained in this manner are far removed from the experimentally measured $\phi$. A third test
TABLE IV. Results in pure compounds. Predictions of scaling relations.

\begin{tabular}{lccc}
\hline \hline Material & $\phi=2 v_{\perp}^{N}-v_{\|}^{N}$ & $\phi=2 v_{\perp}^{\mathrm{Sm}}-v_{\|}^{\mathrm{Sm}}$ & $v_{\|}=1-\alpha / 2-\phi / 2$ \\
\hline 8OCB & $0.45 \pm 0.09$ & $0.96 \pm 0.04$ & $0.73 \pm 0.02$ \\
8CB & $0.35 \pm 0.04$ & & \\
4O.8 & $0.43 \pm 0.02$ & $0.71 \pm 0.06$ & $0.71 \pm 0.03$ \\
8S5 & 0.53 & & $0.78 \pm 0.01$ \\
\hline \hline
\end{tabular}

is obtained by subtracting relation (b) from (a) and obtains $2 v_{\|}=2-\alpha-\phi$; this is evaluated from the measured values of $\alpha$ and $\phi$. The predictions of this text are in poor agreement with the measured values of $v_{\|}^{\mathrm{Sm}}$.

The fact that these measured critical exponents are not universal and do not fit together in a manner that is totally consistent with any of the present theories of the nematic-to-smectic- $\boldsymbol{A}$ phase transition poses a major problem to equilibrium statistical mechanics. One obvious solution to this problem is to assume that the presently measured exponents are effective exponents and that they will evolve into the true exponents describing the asymptotic regime upon examination at smaller $\Delta T$. Should this be the case then we need an argument explaining the fact that the width in temperature of the critical regime only extends to values significantly less than 10 $\mathrm{mK}$. In order to make measurements into this temperature region improvements in temperature control and purity of liquid crystals must be made.

Another solution has been proposed by Nelson and Ton$\mathrm{er}^{11}$ who suggested that in the critical region one should find $2 v_{1}=v_{||}$, and that in the asymptotic limit $B$ is actually independent of $\Delta T$ and nonzero. No experiment to date has obtained $2 v_{1}=v_{\|}$, and none have failed to measure some temperature dependence in $B$. Should this be the case the graphs of the asymptotic behavior of $\log B$ vs $\log \Delta T$ would not be straight lines, as shown in Fig. 7, but would flatten out at $\Delta T \rightarrow 0$. Our data for $80 \mathrm{OB}-6 \mathrm{OCB}$ mixtures ${ }^{38}$ show just such a deviation, and as indicated in Table III the present results are also consistent with a small $B\left(T_{N A}\right) \neq 0$. However, to see a deviation from a simple power law in the present materials much smaller values of $\left(T-T_{N A}\right)$ are required. ${ }^{38}$

\section{ACKNOWLEDGMENTS}

We would like to acknowledge numerous helpful discussions with David Nelson, Bert Halperin, and John Toner. M.R.F. has been the recipient of an IBM Predoctoral Fellowship during a portion of this work. The work was supported by the National Science Foundation under Grant No. DMR-79-23597 and by the Joint Services Electronics Program (U.S. Army, Navy, and Air Force) under Grant No. N00014-75-C-0648. 
*Current address: Physics Department, Massachusetts Institute of Technology, Cambridge, MA 02139.

Current address: Department of Physics, Building FM-15, University of Washington, Seattle, WA 98195.

${ }^{1}$ P. G. de Gennes, Solid State Commun. 10, 753 (1972).

${ }^{2}$ T. C. Lubensky and J.-H. Chen, Phys. Rev. B 17, 366 (1978).

${ }^{3}$ B. I. Halperin and T. C. Lubensky, Solid State Commun. 14, 997 (1974); also see: B. I. Halperin, T. C. Lubensky, and S.K. Ma, Phys. Rev. Lett. 32, 292 (1974); J.-H. Chen, T. C. Lubensky, and D. R. Nelson, Phys. Rev. B 17, 4274 (1978).

${ }^{4}$ C. Dasgupta and B. I. Halperin, Phys. Rev. Lett. 47, 1556 (1981).

${ }^{5} \mathrm{~A}$ review of light scattering experiments through 1978 is given by: R. Schaetzing and J. D. Litster, Advances in Liquid Crystals (Academic, New York, 1979), Vol. 4, p. 147.

${ }^{6}$ J. D. Litster, J. Als-Nielsen, J. R. Birgeneau, S. S. Dana, D. Davidov, F. Garcia-Golding, M. Kaplan, C. R. Safinya, and R. Schaetzing, J. Phys. (Paris) Colloq. 40, C3-339 (1979).

${ }^{7}$ D. Brisbin, R. DeHoff, T. E. Lockhart, and D. L. Johnson, Phys. Rev. Lett. 43, 1171 (1979).

${ }^{8}$ C. W. Garland, M. Meichle, B. M. Ocko, A. R. Kortan, C. R. Safinya, C. J. Yu, J. D. Litster, and R. J. Birgeneau, Phys. Rev. A 27, 3234 (1983).

${ }^{9}$ B. M. Ocko, R. J. Birgeneau, J. D. Litster, and M. E. Neubert, Phys. Rev. Lett. 2, 208 (1984).

10J. Thoen, M. Marynissen, and W. Van Dael, Phys. Rev. Lett. 52, 204 (1984).

${ }^{11}$ D. R. Nelson and J. Toner, Phys. Rev. B 24, 363 (1981).

${ }^{12}$ T. C. Lubensky, S. G. Dunn, and J. Isaacson, Phys. Rev. Lett. 47, 1609 (1981).

${ }^{13}$ T. C. Lubensky J. Chem. Phys. Paris $\underline{80}, 31$ (1983).

${ }^{14}$ See, for instance, H. Birecki, R. Schaetzing, F. Rondelez, and J. D. Litster, Phys. Rev. Lett. 36, 1376 (1976).

${ }^{15}$ N. A. Clark, Phys. Rev. A 14,1551 (1976).

${ }^{16}$ R. Ribbota, C. R. Acad. Sci. Ser. B 279, 295 (1974).

${ }^{17}$ Y. Liao, N. A. Clark, and P. S. Pershan, Phys. Rev. Lett. $\underline{30}$, 639 (1973); also see A. Wergin, W. Krasser, H. H. Stiller, and C. G. B. Frischkorn, Phys. Rev. A 20, 1120 (1979).

${ }^{18}$ S. Battacharya, B. K. Sarma, and J. B. Ketterson, Phys. Rev. B 23, 2397 (1981); also see K. Miyano and J. B. Ketterson,
Physical Acoustics Principles and Methods (Academic, New York, 1979), Vol. 14, p. 93.

${ }^{19}$ B. Y. Cheng, B. K. Sarma, I. D. Calder, S. Battacharya, and J. B. Ketterson, Phys. Rev. Lett. 46, 828 (1981).

${ }^{20}$ L. Ricard and J. Prost, J. Phys. (Paris) 42, 861 (1981).

${ }^{21}$ M. R. Fisch, L. B. Sorensen, and P. S. Pershan, Mol. Cryst. Liq. Cryst. 67, 165 (1981).

${ }^{22}$ M. R. Fisch, L. B. Sorensen, and P. S. Pershan, Phys. Rev. Lett. 47, 43 (1981).

${ }^{23}$ P. S. Pershan, J. Appl. Phys. 45, 1590 (1974).

24J. Als-Nielsen, F. Christensen, and P. S. Pershan, Phys. Rev. Lett. $\underline{48}, 1107$ (1982).

${ }^{25}$ M. R. Fisch, Ph.D. dissertation, Harvard University, 1981 (unpublished).

26P. G. de Gennes, J. Phys. (Paris) Colloq. 30, C4-65 (1969).

${ }^{27}$ See, for instance, L. D. Landau and E. M. Lifshitz, Fluid Mechanics (Pergamon, New York, 1975), pp. 5 and 50.

${ }^{28}$ Orsay Group on Liquid Crystals, J. Phys. (Paris) Colloq. $\underline{36}$, C1-305 (1975).

${ }^{29}$ C. H. Sohl, K. Miyano, and J. B. Ketterson, Rev. Sci. Instrum. 49, 1464 (1978).

${ }^{30}$ S. Hard, Y. Hammerius, and D. Nilsson, J. Appl. Phys. 47, 2433 (1976).

${ }^{31}$ F. R. Bouchet and P. E. Cladis, Mol. Cryst. Liq. Cryst. Lett. 64, 81 (1980).

${ }^{32}$ A. J. Leadbetter, J. L. A. Durrant, and M. Rugman, Mol. Cryst. Liq. Cryst. Lett. 34, 231 (1977).

${ }^{33}$ P. P. Karat and N. V. Madhusudama, Mol. Cryst. Liq. Cryst. 47, 21 (1978).

${ }^{34}$ R. J. Birgeneau, C. W. Garland, G. B. Kasting, and B. M. Ocko, Phys. Rev. A 24 2624 (1981).

${ }^{35}$ H. von Kanel and J. D. Litster, Phys. Rev. A 233, 3251 (1981).

${ }^{36}$ C. R. Safinya, R. J. Birgeneau, J. D. Litster, and M. E. Neubert, Phys. Rev. Lett. 느, 668 (1981).

${ }^{37}$ C. W. Garland, G. B. Kasting, and K. J. Lushington, Phys. Rev. Lett. 43, 1420 (1979); G. B. Kasting, K. J. Lushington, and C. W. Garland, Phys. Rev. B 22, 321 (1980); also see C. A. Schantz and D. L. Johnson, Phys. Rev. A 17, 1504 (1978).

${ }^{38}$ M. R. Fisch, L. B. Sorensen, and P. S. Pershan, Phys. Rev. Lett. 48,943 (1982). 